\title{
Disposición espacial de parches de Austrocedrus chilensis con síntomas de defoliación y mortalidad en el Valle 16 de Octubre (Chubut, Argentina)
}

\author{
Spatial distribution of Austrocedrus chilensis patches with defoliation \\ and mortality in Valle 16 de Octubre, Chubut province, Argentine
}

\author{
Ludmila La Manna ${ }^{a, b, c *}$, Francisco Carabellic, Mariano Gómez ${ }^{a}$, c, Silvia D. Matteuccib, d \\ *Autor de correspondencia: ${ }^{a}$ Centro de Investigación y Extensión Forestal Andino \\ Patagónico, C.C. 14 (9200), Esquel, Chubut, Argentina, llamanna@ciefap.org.ar

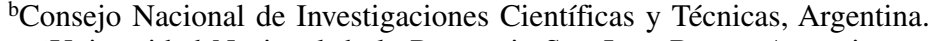 \\ cUniversidad Nacional de la Patagonia San Juan Bosco, Argentina. \\ dUniversidad de Buenos Aires, Grupo de Ecología del Paisaje y Medio Ambiente, Argentina.
}

\begin{abstract}
SUMMARY
Cordilleran cypress (Austrocedrus chilensis) is an endemic species of the Andean forests of Patagonia. Widespread mortality of A. chilensis occurs and is locally known as "mal del ciprés". The objectives of this work were to identify the spatial distribution of patches presenting mal del ciprés syndrome and to quantify the surface showing affected forest. The study was carried out in " 16 de Octubre" Valley (Chubut province, $43^{\circ} 10^{\prime}$ 'S), which concentrates $9 \%$ of the total surface of A. chilensis pure forests in Argentina. In the field, patches of trees affected by "mal del ciprés" (100), patches of trees in asymptomatic dense forests (50) and patches of trees in asymptomatic thin forests (80) were identified. According to these training sites, the digital numbers for each type of vegetation were determined and a supervised classification was done using SPOT PAN and XS satellite images. Discrimination between "mal del ciprés" and asymptomatic thin patches was hard because both types of vegetation presented similar digital numbers, probably because of the understory influence. Classification accuracy was assessed in the field. In the final map, 70\% of patches with "mal del ciprés" were well classified. Eighty five percent of patches with asymptomatic dense forest and $70 \%$ of patches with asymptomatic thin forests were well classified. The affected forest covered 1,678 ha (24\% of the total surface of A. chilensis in the study area). The asymptomatic dense forest and asymptomatic thin forest covered 2,721 and 2,584 ha, respectively. This study allowed quantifying the magnitude of mal del ciprés and the generated map could be useful for epidemic studies and for forest management.
\end{abstract}

Key words: cordilleran cypress, "mal del ciprés”, classification, mapping.

\section{RESUMEN}

El ciprés de la cordillera (Austrocedrus chilensis), especie endémica de los bosques andinopatagónicos, sufre una enfermedad conocida como "mal del ciprés". Los objetivos de este trabajo fueron identificar la disposición espacial de parches de A. chilensis con síntomas

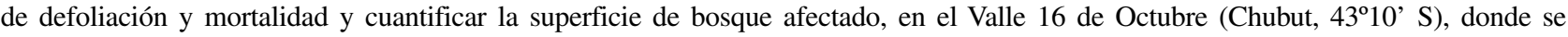
concentra el 9\% de la superficie total de los bosques puros de A. chilensis en Argentina. En el campo se identificaron grupos de árboles afectados por el mal del ciprés (100), asintomáticos en áreas de bosque denso (50) y en áreas de bosque ralo (80). Con estas áreas de entrenamiento se determinaron los niveles digitales según tipo de vegetación y se realizó una clasificación supervisada utilizando imágenes satelitales SPOT PAN y XS. Resultó dificultosa la discriminación entre mal del ciprés y bosque asintomático ralo, porque ambos presentaron niveles digitales similares, posiblemente por influencia del sotobosque. Del mapa obtenido fueron bien clasificados el 70\% de los parches con mal del ciprés, el $85 \%$ de los parches densos asintomáticos y el $70 \%$ de los parches ralos asintomáticos. El bosque afectado abarcó 1.678 ha (24\% de la superficie total de A. chilensis en el área de estudio) y los bosques asintomático denso y ralo ocuparon 2.721 y 2.584 ha, respectivamente. Este estudio permitió cuantificar, por primera vez, la magnitud del mal del ciprés y el mapa generado constituye una herramienta útil para estudios epidemiológicos y de manejo del bosque.

Palabras clave: ciprés de la cordillera, "mal del ciprés", clasificación, mapeo.

\section{INTRODUCCIÓN}

El ciprés de la cordillera [Austrocedrus chilensis (D. Don) Pic. Serm. et Bizarri] es una especie endémica de los bosques andinopatagónicos. Los bosques de A. chilensis presentan su mayor desarrollo en la vertiente oriental de
Los Andes, en Argentina, cubriendo aproximadamente 141.000 ha (Bran et al. 2002). Se ubican en el piedemonte cordillerano entre los $37^{\circ} 7^{\prime}$ y $39^{\circ} 30^{\prime}$ 'S en forma discontinua y, en forma más continua, entre los $39^{\circ} 30^{\prime}$ y $43^{\circ} 44^{\prime} \mathrm{S}$, en una franja de hasta $60-80 \mathrm{~km}$ de ancho, que incluye un fuerte gradiente pluviométrico (Bran et al. 2002, Pastorino 
et al. 2006). Hacia el Oeste, en sitios con precipitaciones entre 1.600 y $2.000 \mathrm{~mm}$, forma bosques mixtos con especies del género Nothofagus (Dezzotti y Sancholuz 1991). En sitios de entre 1.600 y $600 \mathrm{~mm}$ de precipitación anual A. chilensis forma bosques monoespecíficos de distintas densidades. Con la disminución de la precipitación hacia el Este, el bosque se torna cada vez más abierto, primero en forma de bosquetes aislados en el ecotono bosque-estepa $\mathrm{y}$, finalmente, con individuos aislados en una matriz de estepa (Veblen et al. 1996).

Los bosques de A. chilensis pueden ser de estructura regular o irregular en cuanto a la distribución de edades. En sitios húmedos, luego de incendios y a partir de árboles sobrevivientes, se establece, por semilla, una regeneración densa y homogénea generando una estructura regular, es decir, todos los árboles son coetáneos. Sin embargo, estos bosques son usualmente de estructura irregular en cuanto a la distribución de frecuencias diamétricas (Bava y Gonda 1993). En sitios secos, el proceso de establecimiento de la regeneración presenta una dinámica diferente, donde la protección que generan los arbustos y los cipreses establecidos previamente juega un rol fundamental. En sitios secos se forman estructuras irregulares tanto en la distribución de edades como en la distribución de frecuencias diamétricas (Loguercio et al. 1999).

Estos bosques constituyen un importante recurso económico para la región cordillerana. La distribución de $A$. chilensis se superpone con los asentamientos urbanos y periurbanos al pie de la cordillera. Estos bosques cumplen así un rol ambiental, paisajístico y recreativo, y constituyen una fuente esencial de servicios ecosistémicos. Desde el punto de vista maderero, $A$. chilensis es la segunda especie nativa en importancia forestal luego de la lenga [Nothofagus pumilio (Poepp. et Endl.) Krass] (SRNyAH 1994). Su madera es noble, de alta calidad y buena forma, apta para múltiples usos (construcción, mueblería, artesanías, etc.) (Díaz-Vaz 1985).

Los bosques de $A$. chilensis son sometidos a la extracción de madera y leña, a una fuerte presión de herbivoria (Veblen et al. 1995, Relva y Veblen 1998) y a frecuentes incendios, generalmente de origen antrópico (Loguercio et al. 1999). Uno de los principales disturbios que afecta a los bosques de $A$. chilensis es la existencia de mortandad masiva de árboles, conocida como "mal del ciprés", que actualmente se extiende por toda el área de distribución de la especie en Argentina. La sintomatología de esta enfermedad se manifiesta como un decaimiento progresivo de los árboles afectados, a lo largo de varios años. Se forman manchones más o menos extendidos que involucran desde pocos árboles (4-5) hasta superficies afectadas de dos hectáreas (Varsavsky et al. 1975). En los individuos afectados ocurre una defoliación progresiva, uniforme o no, que va dejando a la copa con menor cantidad de hojas. Los síntomas foliares aparecen inicialmente como un amarillamiento, luego amarronamiento y, finalmente, la caída foliar. La distribución y el avance de la defoliación responden a diferentes patrones y fueron caracterizados por Havrylenko et al. (1989). El decaimiento se manifiesta también con una disminución del crecimiento radial (Calí 1996), el progresivo deterioro de las raíces y el desarrollo de pudriciones en las raíces principales (Barroetaveña y Rajchenberg 1996).

Los bosques de A. chilensis presentan diferentes estructuras en función de la ocupación del sitio y la altura de los árboles dominantes. Estas estructuras se asocian con distintos tipos de suelo, y el mal del ciprés no está restringido a una estructura en particular (La Manna et al. 2006). Si bien a priori podría suponerse que como consecuencia de la enfermedad se generan claros, aumentando la entrada de luz y creando condiciones de regeneración, la enfermedad está negativamente asociada con la abundancia de renovales de $A$. chilensis, al menos en bosques sometidos a uso ganadero (La Manna et al. en prensa).

Numerosos estudios han sido realizados con el fin de determinar la causa de la enfermedad (Barroetaveña y Rajchenberg 1996, Rajchenberg et al. 1998, Greslebin et al. 2004, 2005, El Mujtar y Andenmatten 2007). Recientemente, y basándose en técnicas moleculares, se ha podido identificar el agente biológico responsable del mal del ciprés. El agente causal es un hongo del género Phytophthora (Phytophthora austrocedrae Gresl. et EM Hansen, sp. nov.), que ha sido recientemente aislado y descrito (Greslebin et al. 2007) y cumple los postulados de Koch (Greslebin y Hansen 2007). El género Phytophthora pertenece al grupo de los mohos acuáticos y su medio de dispersión es a través de esporas flageladas que requieren de agua para su movilidad. Por otro lado, estudios realizados en bosques de $A$. chilensis de Chile hallaron al pulgón Cinara cupressi (Homoptera: Aphididae) como agente responsable en la sintomatología de defoliación y mortalidad del bosque (Baldini et al. 2004). Sin embargo, estos estudios no evaluaron el estado del sistema radical. El mal del ciprés también ha sido asociado a factores abióticos, como suelos de drenaje impedido (La Manna y Rajchenberg 2004ab) y zonas con altas precipitaciones y altitudes medias (Baccalá et al. 1998).

Si bien existen registros puntuales de la enfermedad en diferentes zonas del área de distribución de A. chilensis en Argentina (Hranilovic 1988, Havrylenko et al. 1989, Rosso et al. 1994, La Manna y Rajchenberg 2004ab, La Manna et al. 2006), no existen mapas de la distribución del mal del ciprés y se desconoce su magnitud. Para este tipo de estudios de vegetación la teledetección es una técnica cada vez más aplicada, dado que reduce el costo y el tiempo invertido en la interpretación de las cubiertas forestales. Los sensores de alta resolución, como los montados en el SPOT 4, con resolución espacial de $20 \mathrm{~m}$ en multiespectral y de $10 \mathrm{~m}$ en pancromática, permitieron superar algunas de las limitaciones de los anteriores sensores (Karszenbaum 1998, Cano et al. 2005). Las imágenes obtenidas mediante el satélite SPOT han sido utilizadas para estudios de vegetación (Pisek et al. 2007) y estudios de evaluación de 
defoliación y daño por plagas (Joria et al. 1991, Brockhaus et al. 1992, Fraser y Latifovic 2005). En Patagonia, este tipo de imágenes fueron utilizadas en estudios de cobertura de especies de Nothofagus (Lencinas y Farías 2005) y para estudios de fragmentación de bosques de A. chilensis (Carabelli 2004, Carabelli y Claverie 2005).

Contar con una metodología de teledetección que permita evaluar la defoliación y mortalidad en los bosques de A. chilensis constituiría una herramienta relevante para estudios de patología forestal y de progresión del disturbio. A su vez, contar con un mapa de la disposición espacial de los parches con síntomas de mal del ciprés permite, mediante la aplicación de sistemas de información geográfica, evaluar la asociación entre la enfermedad y variables climáticas, geomórficas y/o edáficas, y en base a esto, generar mapas de riesgo de ocurrencia (Kaya et al. 2002, Meentemeyer et al. 2004, Van Staden 2004, Allen y Wong 2006, Fernández y Solla 2006), herramienta fundamental para determinar pautas de manejo de estos bosques.

Los objetivos de este trabajo fueron a) identificar la disposición espacial de parches de A. chilensis con síntomas de defoliación y mortalidad en el Valle 16 de Octubre, provincia del Chubut, y b) cuantificar la superficie de bosque afectado por mal del ciprés en dicho Valle.

\section{MÉTODOS}

El estudio se centró en el área del Valle 16 de Octubre, provincia del Chubut ( $\left.43^{\circ} 10^{\prime} \mathrm{S}\right)$, donde A. chilensis forma bosques puros. Constituye un área de interés dado que representa el $9 \%$ de la superficie total de los bosques puros de A. chilensis en Argentina (Carabelli y Claverie 2005). $\mathrm{El}$ área de estudio incluye un fuerte gradiente pluviométrico Este-Oeste, con valores desde 600 a $1.300 \mathrm{~mm}$ de precipitación anual, con diferentes geomorfologías (Rabassa et al. 2001) y tipos de suelo (La Manna 2005).

En el campo se identificaron 100 grupos de árboles (parches) con síntomas externos de mal del ciprés (amarillamiento o defoliación parcial o total). Se identificaron también 50 grupos de árboles asintomáticos en áreas de bosque denso y 80 en áreas de bosque ralo. Mediante un sistema de posicionamiento global (GPS) Trimble se determinó la ubicación y perímetro de cada grupo de árboles identificado. El criterio para la selección de los sitios de muestreo fue que el grupo de árboles muestreado presentara un tamaño mínimo de $400 \mathrm{~m}^{2}$ y tuviera un sector de borde (por fuera del polígono muestreado) de al menos 10 metros con un estado sanitario similar al interior. A fin de que el muestreo fuera representativo se abarcaron las distintas situaciones de precipitación, geomorfología y suelos del área de estudio (Rabassa et al. 2001, La Manna 2005). Se realizó una caracterización general de la vegetación del parche, incluyendo el número de árboles asintomáticos, sintomáticos y muertos, se evaluó visualmente la cobertura de los estratos herbáceo y arbustivo del sotobosque y se registraron las especies dominantes.

En gabinete se trabajó con imágenes satelitales SPOT PAN y XS georreferenciadas del año 2001, con una resolución espacial de $10 \mathrm{~m}$, abarcando un área de 30 x 30 $\mathrm{km}$. Estas imágenes fueron previamente ortorrectificadas y corregidas en el marco del proyecto Change on landscape heterogeneity of 'ciprés de la cordillera' forests (Carabelli et al. 2003). Los datos obtenidos con el GPS en el muestreo de campo fueron transformados al sistema de coordenadas proyectadas Argentina Faja 1, Datum Campo Inchauspe mediante el programa GPS Pathfinder. En base a los polígonos de campo (áreas de entrenamiento) se determinaron los niveles digitales medios para cada tipo de vegetación muestreado: A. chilensis asintomático denso, A. chilensis asintomático ralo y $A$. chilensis afectado por mal del ciprés. Los niveles digitales, es decir los valores que presentan los píxeles en la imagen, se encuentran entre 0 y 255 dado que el intervalo dinámico de los sensores SPOT se discretiza en 256 niveles ( 0 a 255), lo que involucra datos de 8 bits (Karszenbaum 1998). La diferencia entre los niveles digitales para cada tipo de vegetación fue evaluada, para cada banda de la imagen, mediante análisis de varianza de un factor y posterior análisis de comparaciones múltiples de Dunnett, el cual no asume homogeneidad de varianzas (Ramsey y Schafer 1997). Se evaluó también la diferencia entre los niveles digitales de las áreas de entrenamiento de mal del ciprés con distintos grados de incidencia de la enfermedad (todos los individuos con defoliación parcial o total vs. mezcla de individuos sintomáticos y asintomáticos) y con distintas coberturas de sotobosque (menos del $60 \%$ de cobertura de herbáceas vs. más del 60\%). Las diferencias fueron analizadas para cada banda mediante la prueba de t. Se evaluó también la distribución de los niveles digitales para cada tipo de vegetación mediante la construcción de histogramas.

Con base en los valores de niveles digitales de los sitios de entrenamiento se realizó una clasificación supervisada de la imagen SPOT combinando las cuatro bandas, mediante el módulo Signature Editor del software ERDAS Imagine. Dado que se contaba con una cobertura preexistente de la distribución de A. chilensis en el área de estudio (Carabelli 2004, Carabelli y Claverie 2005), la clasificación supervisada se limitó al área ocupada por bosques de $A$. chilensis mediante la aplicación "área de interés" (AOI) del software ERDAS Imagine.

La clasificación fue sucesivamente depurada de acuerdo con cuatro consideraciones: a) Se realizó una recodificación de la imagen clasificada, incluyendo tres clases: "mal del ciprés", "A. chilensis asintomático denso" y "A. chilensis asintomático ralo". b) Se aplicó un algoritmo que identifica conglomerados (grupo de celdas contiguas) a base de los ocho vecinos conectados de una misma clase temática (función clump del programa ERDAS Imagine). c) Posteriormente se utilizó la función eliminate para excluir los conglomerados menores a la unidad mínima 
cartografiable (SAyDS 2005). d) La imagen clasificada fue luego convertida a formato vector, primero como archivo arcinfo y, posteriormente, como cobertura shape.

A partir de esta cobertura se generó una cobertura de A. chilensis asintomático denso, eliminando todos los polígonos que no habían sido clasificados en esta categoría, y donde se eliminaron también los polígonos con superficie menor a $900 \mathrm{~m}^{2}$, siguiendo la metodología propuesta por Carabelli (2004) y Carabelli y Claverie (2005).

Se generó también una cobertura de A. chilensis asintomático ralo y mal del ciprés, a la cual se le restaron los polígonos con superficie menor a $400 \mathrm{~m}^{2}$, que corresponde a la superficie mínima de las áreas de entrenamiento. Esta cobertura se utilizó para generar una nueva área de interés (AOI). En base a los resultados del análisis de varianza, para discriminar A. chilensis asintomático ralo de mal del ciprés se realizó una segunda clasificación supervisada de la imagen SPOT considerando únicamente la banda 4 (infrarrojo medio). Para esta clasificación se utilizaron los niveles digitales de las áreas de entrenamiento correspondientes a mal del ciprés y A. chilensis asintomático ralo, y se limitó al área de interés generada. Esta clasificación fue sucesivamente depurada siguiendo las operaciones espaciales descritas previamente (SAyDS 2005), generándose una cobertura de A. chilensis ralo y otra de mal del ciprés.

Se obtuvo así un mapa preliminar de la distribución de tres tipos de vegetación considerados: mal del ciprés, $A$. chilensis asintomático denso y A. chilensis asintomático ralo. En base a esta cobertura se seleccionaron al azar 200 puntos de control, que fueron ingresados al GPS, y en el campo se evaluó la correspondencia entre el tipo de vegetación clasificado en el mapa y la vegetación real. Posteriormente, se hicieron recorridos de toda el área de estudio, utilizando caminos principales y secundarios, evaluando nuevamente la correspondencia entre el tipo de vegetación clasificado en el mapa y la vegetación real. Para esta tarea el mapa generado fue impreso sobre las cartas del Instituto Geográfico Militar (IGM) digitalizadas a fin de poder ubicarse en el terreno. Esta información de campo fue utilizada para depurar el mapa preliminar, corrigiendo las coberturas correspondientes a los distintos tipos de vegetación. Se obtuvo así un mapa definitivo con las coberturas de mal del ciprés, $A$. chilensis asintomático denso y A. chilensis asintomático ralo. A fin de validar el mapa final se seleccionaron al azar 100 puntos de control, que fueron ingresados al GPS, y en el campo se evaluó la correspondencia entre el tipo de vegetación clasificado en el mapa temático y la vegetación real. A partir de esta evaluación se determinó el porcentaje de casos correctamente clasificados como un estimador de la exactitud del mapa temático (Smits et al. 1999, Foody 2002).

Para el procesamiento se utilizó el software ERDAS Imagine 8.4 y para la generación de las coberturas temáticas el software ArcView 3.2.

\section{RESULTADOS}

Todos los bosques correspondieron a bosques puros de A. chilensis. Lomatia hirsuta (Lam.) Diels [radal] estuvo presente como especie acompañante en la mayoría de los sitios muestreados, tanto asintomáticos como afectados, y en algunas zonas también Maytenus boaria (Mol.) [maitén]. Ejemplares de Nothofagus antarctica (Forst.) Oerst [ñirre] y Nothofagus dombeyi (Mirb. Oerst.) [coihue] fueron raramente registrados.

Tanto en parches asintomáticos como afectados la cobertura del estrato arbustivo fue muy variable, desde áreas prácticamente sin arbustos hasta un $80 \%$ de cobertura. El estrato arbustivo bajo estuvo dominado por Aristotelia chilensis L'Hér. Stirp [maqui] y Schinus patagonicus (Phil.) Johnst. [laura]. En algunas zonas se registraron Rosa eglanteria L. [rosa mosqueta], Maytenus chubutensis (Speg.) Lourt., O'Don. et Sleum [chaurilla] y distintas especies del género Berberis. La cobertura del estrato herbáceo alcanzó valores de hasta 95\%. Los ejemplares dominantes del estrato herbáceo correspondieron principalmente a distintas especies de gramíneas. Fueron frecuentes también Acaena ovalifolia Ruiz y Pavón y Acaena pinnatifida Ruiz y Pavón. Se registraron frecuentemente helechos de la especie Ruhmora adiantiformis (Forst.) Ching.

Los grupos de árboles afectados muestreados presentaron diferencias en los niveles de incidencia del mal del ciprés, desde parches con todos los individuos muertos, parches con mayoría de árboles con síntomas de defoliación, hasta grupos de árboles con individuos de distinta vitalidad entremezclados (asintomáticos, sintomáticos y muertos) (La Manna y Carabelli 2005).

Esta variación en los niveles de incidencia de la enfermedad y en las características del sotobosque se refleja en los valores que toman los píxeles en la imagen satelital al evaluar áreas representativas de bosque con mal del ciprés. Al comparar los niveles digitales de las áreas de entrenamiento de mal del ciprés que presentaron todos los individuos afectados (defoliación parcial o total) con aquellas áreas de entrenamiento que presentaron mezclas de vitalidades, incluyendo árboles asintomáticos, se halló que los valores difieren significativamente entre ambos grupos para la banda 2, correspondiente a la banda de absorción de clorofila (rojo) (Karszeunbaum 1998). A su vez, al comparar los niveles digitales de las áreas de entrenamiento de mal del ciprés que presentaron menos del $60 \%$ de cobertura de herbáceas con aquellas que presentaron más del $60 \%$, se hallaron diferencias significativas $(P<0,05)$ para las cuatro bandas de la imagen.

Las características intrínsecas del bosque de $A$. chilensis, con grandes variaciones en la cobertura de sotobosque y diferencias en los niveles de incidencia de la enfermedad, determinaron un amplio espectro de niveles digitales en las distintas bandas para los tres tipos de vegetación considerados, especialmente para A. chilensis asintomático ralo y mal del ciprés (figura 1 ). 

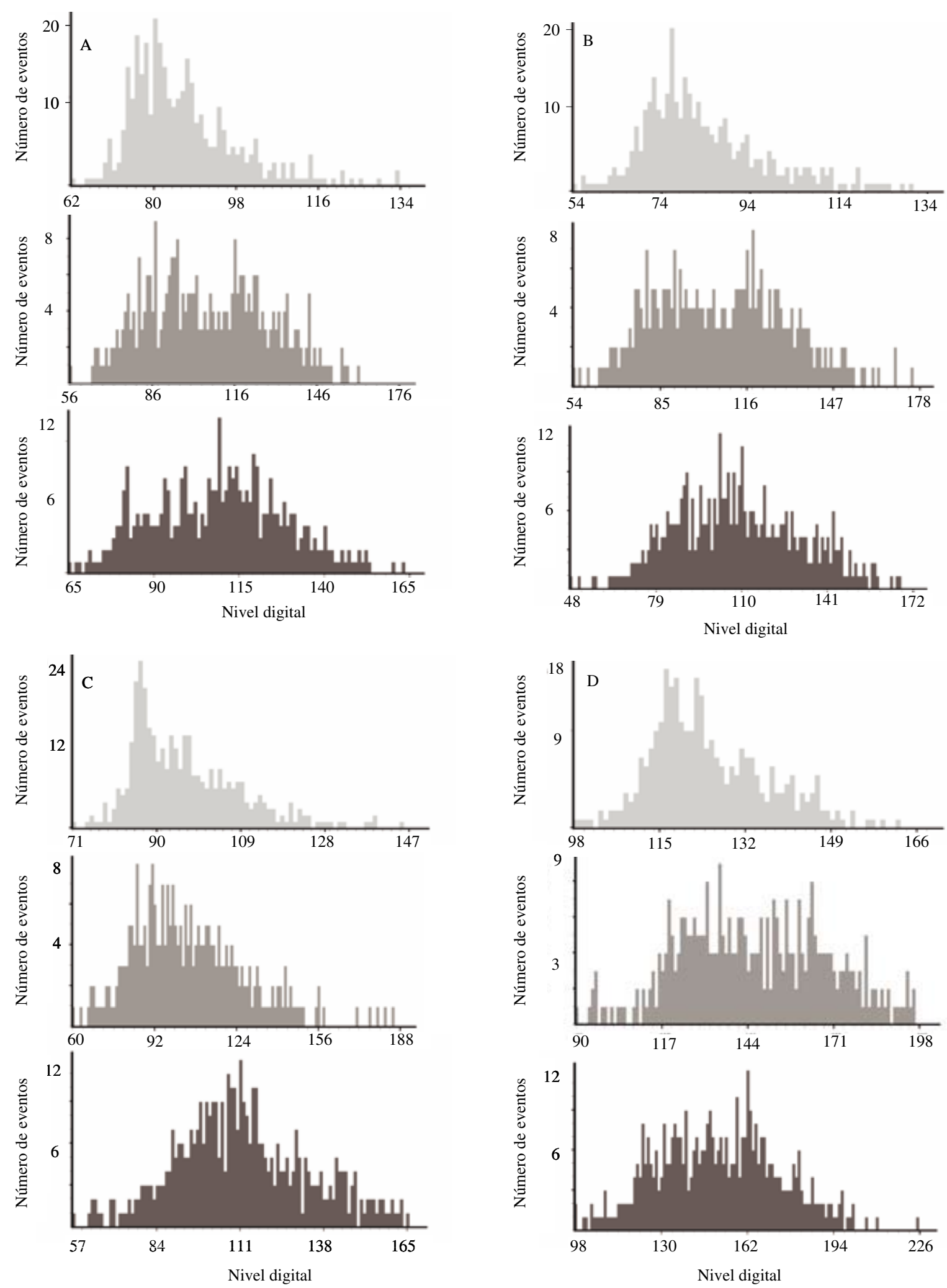

Figura 1. Distribución de los niveles digitales para las bandas 1 (A), 2 (B), 3 (C) y 4 (D) de una imagen SPOT correspondientes a píxeles representativos de áreas con vegetación de: $\square$ Austrocedrus chilensis asintomático denso, $\square$ A. chilensis asintomático ralo, $\square$ mal del ciprés.

Distribution of digital numbers for band 1 (A), 2 (B), 3 (C) and 4 (D) of the SPOT image corresponding to representative pixels from areas with dense asymptomatic A. chilensis, thin asymptomatic A. chilensis, A. chilensis affected by "mal del ciprés". 
Los niveles digitales correspondientes a A. chilensis asintomático denso fueron significativamente menores $(P<0,05)$ a los de mal del ciprés y A. chilensis asintomático ralo para las cuatro bandas de la imagen SPOT (figura 2). Por el contrario, los niveles digitales correspondientes a $A$. chilensis asintomático ralo y mal del ciprés fueron similares para las distintas bandas y únicamente en la banda 4, correspondiente al infrarrojo medio o de onda corta, presentaron una diferencia significativa $(P<0,05)$ de acuerdo al análisis de varianza (figura 2). Estos resultados determinaron la necesidad de hacer una primera clasificación combinando las cuatro bandas y una segunda clasificación únicamente con la banda 4 para discriminar A. chilensis asintomático ralo de mal del ciprés.

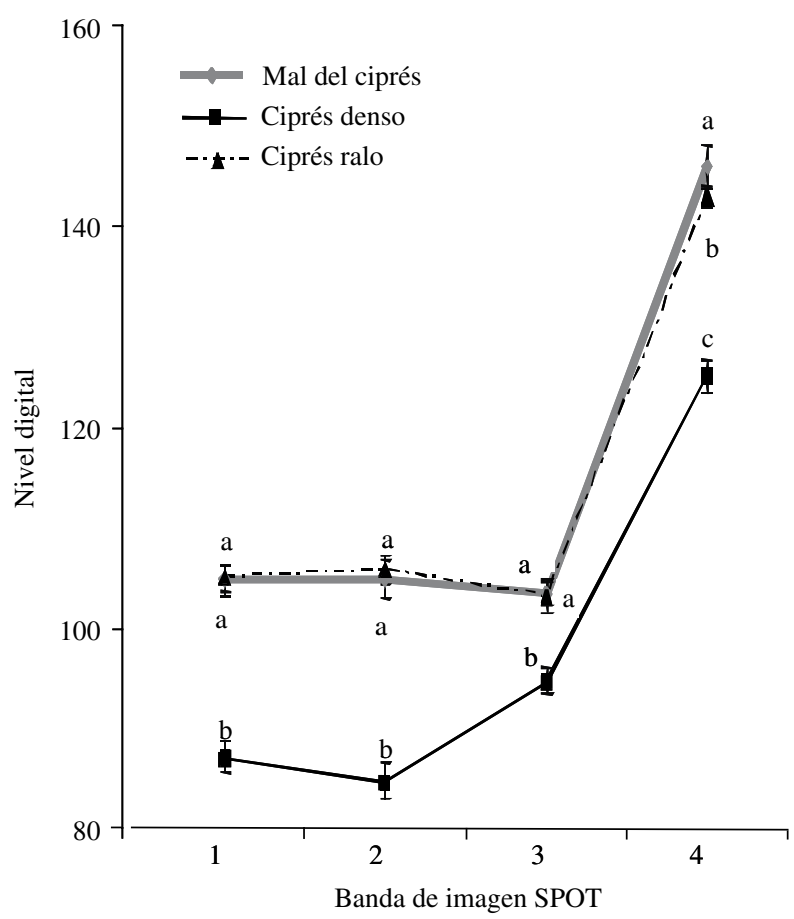

Figura 2. Números digitales medios ( \pm 1 desvío estándar) de parches con tres tipos de vegetación de A. chilensis: bosque con síntomas de mal del ciprés, bosque asintomático denso, bosque asintomático ralo, para las cuatro bandas de una imagen SPOT. Letras distintas indican diferencias significativas entre los tipos de vegetación dentro de una misma banda.

Digital numbers mean values ( \pm 1 standard error) of patches with three vegetation types of Austrocedrus chilensis: forest affected by "mal del ciprés", dense asymptomatic forest, thin asymptomatic forest, for the four bands of an SPOT image. Different letters indicate significant differences between the vegetation types for each band.

La dificultad para discriminar A. chilensis asintomático ralo de mal del ciprés, sumado al amplio espectro de niveles digitales hallados, conllevó la exigencia de efectuar un intenso control de terreno para ajustar la distribución de las distintas coberturas. El mapa preliminar generado presentó un alto error, asociado con dos situaciones: los bosques asintomáticos ralos fueron frecuentemente clasificados como sintomáticos; y ciertos parches de árboles sintomáticos con una vegetación arbustiva densa fueron clasificados como asintomáticos. Esta situación conllevó la exigencia de reforzar el trabajo de campo y corregir las coberturas obtenidas a partir del análisis de la correspondencia entre la clasificación supervisada de gabinete y los sectores efectivamente sintomáticos y asintomáticos. Para poder generar un mapa fiable debió recurrirse a una técnica alternativa, utilizando un método iterativo entre procesamiento de imagen y chequeo a campo.

El último control de campo, realizado según el mapa final, determinó que el $70 \%$ de los parches con mal del ciprés fueron bien clasificados. A su vez, el $85 \%$ de los parches densos de A. chilensis asintomático y el $70 \%$ de los parches ralos asintomáticos fueron bien clasificados.

Los resultados de la clasificación de los parches con distintos tipos de vegetación indican que el bosque con síntomas de mal del ciprés abarcó una superficie de 1.678 ha, en tanto el bosque de A. chilensis asintomático denso y ralo ocuparon una superficie de 2.721 y 2.584 ha, respectivamente (figura 3, cuadro 1). El área afectada equivale así al $24 \%$ de la superficie total de A. chilensis en el área de estudio.

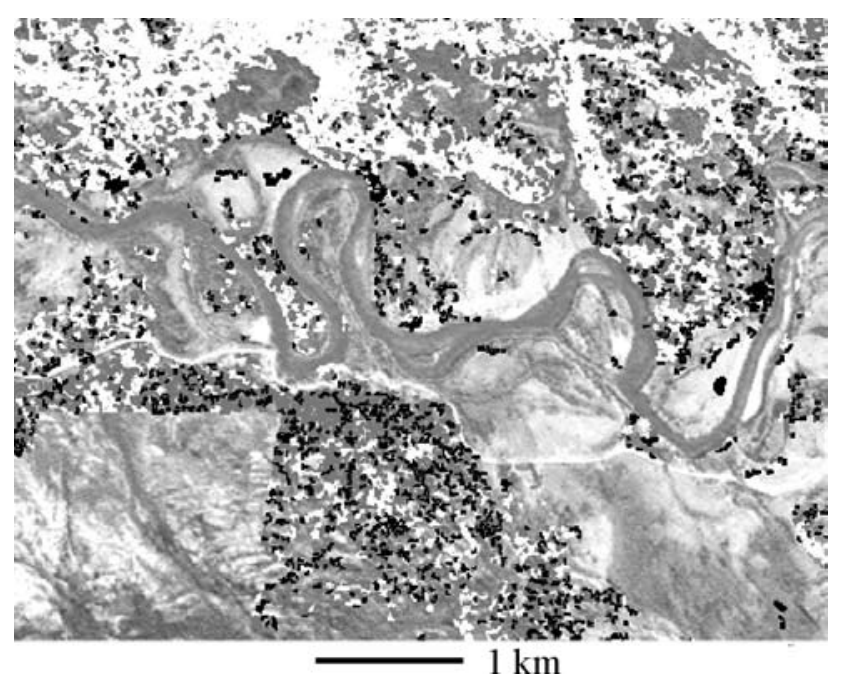

Figura 3. Distribución de distintos tipos de vegetación de Austrocedrus chilensis en un sector del Valle 16 de Octubre (Provincia de Chubut, Patagonia, Argentina) sobre una imagen SPOT: bosque afectado por mal del ciprés (en negro), bosque asintomático denso (blanco) y bosque asintomático ralo (gris).

Spatial distribution of patches with different Austrocedrus chilensis vegetation types in a sector of 16 de Octubre Valley (Chubut province, Argentine Patagonia) on a SPOT image: forests affected by "mal del ciprés" (black), dense asymptomatic forest (white) and thin asymptomatic forest (grey). 
Cuadro 1. Valores de cantidad de parches, superficies y desvío estándar de las coberturas de la distribución de Austrocedrus chilensis asintomático denso, A. chilensis asintomático ralo y mal del ciprés en el Valle 16 de Octubre (Chubut).

Number of patches, surface and standard deviation for the spatial distribution of dense asymptomatic A. chilensis, thin asymptomatic A. chilensis and A. chilensis affected by "mal del ciprés" in 16 de Octubre Valley (Chubut).

\begin{tabular}{lcccc}
\hline \multirow{2}{*}{ Nombre cobertura } & \multirow{2}{*}{\begin{tabular}{c}
$\mathrm{N}^{\mathrm{o}}$ \\
\cline { 3 - 5 }
\end{tabular}} & \multicolumn{3}{c}{ Superficie (ha) } \\
\cline { 3 - 5 } & 4.263 & $0,64 \pm 5,66$ & $0,09-249,5$ & 2.721 \\
\hline $\begin{array}{l}\text { A. chilensis } \\
\text { asintomático denso }\end{array}$ & & & & \\
$\begin{array}{l}\text { A. chilensis } \\
\text { asintomático ralo }\end{array}$ & 15.013 & $0,17 \pm 0,39$ & $0,04-20,85$ & 2.584 \\
\begin{tabular}{l} 
Mal del ciprés \\
\hline
\end{tabular} & 16.680 & $0,10 \pm 0,14$ & $0,04-7,79$ & 1.678 \\
\hline
\end{tabular}

\section{DISCUSIÓN}

Los bosques de $A$. chilensis presentan una gran variación en cobertura de sotobosque y en los niveles de incidencia de la enfermedad, lo cual representó una dificultad para estimar la sanidad del bosque mediante técnicas de sensores remotos. Los tres tipos de vegetación considerados en este estudio (mal del ciprés, A. chilensis asintomático denso y $A$. chilensis asintomático ralo) evidenciaron un amplio espectro de niveles digitales, es decir, del valor que toman los píxeles en la imagen satelital. El rango de valores fue amplio para las cuatro bandas que considera la imagen, en especial para A. chilensis asintomático ralo y mal del ciprés. Sin embargo, existió una clara diferenciación de bosque asintomático denso y mal del ciprés, presentando el bosque denso niveles digitales significativamente menores que el bosque sintomático para las distintas bandas. Los niveles digitales guardan una relación positiva con los valores de reflectancia, es decir, que el bosque asintomático denso presenta bajos valores de reflectancia en comparación con el bosque sintomático.

La distinción entre mal del ciprés y A. chilensis asintomático ralo fue dificultosa y ambos tipos de vegetación presentaron un intervalo muy amplio de niveles digitales, posiblemente por la influencia de la vegetación de sotobosque. En un caso, porque los árboles afectados presentan disminución o falta total de follaje, y en el caso del bosque ralo porque se trata de bosques abiertos donde adquiere predominancia la reflectancia del sotobosque. Los niveles digitales fueron similares entre ambos tipos de vegetación para las tres bandas del espectro visible y únicamente se evidenciaron diferencias significativas en el infrarrojo medio (banda 4 de la imagen Spot). En el infrarrojo medio existe un pico de absorción, de modo que el grado de hidratación de los tejidos vegetales influye fuertemente en la cantidad de luz reflejada en esta porción del espectro (Towers y von Martini 2002). Así, los parches con mal del ciprés, donde el follaje está seco, evidenciaron valores significativamente mayores de nivel digital que el bosque asintomático ralo.

En un estudio similar en bosques montanos de Carolina del Norte se encontraron diferencias en los niveles digitales en el infrarrojo cercano en función del grado de defoliación de los árboles. Sin embargo, ninguno de los sensores utilizados (SPOT HRV y Landsat TM) fueron buenos predictores del grado de defoliación en ecuaciones de regresión (Brockhaus et al. 1992). Si bien existen numerosos antecedentes en el empleo de sensores remotos para el estudio de plagas y enfermedades, en general éstos han sido útiles para cultivos anuales dado que las plantas son atacadas en masa, en grandes superficies y su aspecto se diferencia claramente en el periodo de crecimiento (Shurtleff y Averre III 1997). En general, el mapeo de bosques con síntomas de defoliación y mortalidad es dificultoso, dadas las características intrínsecas del sistema boscoso. Aun cuando la defoliación causada por insectos ha sido frecuentemente evaluada mediante técnicas de teledetección, las clasificaciones de defoliación de áreas boscosas han sido sólo moderadamente exitosas, y continúa siendo una necesidad mejorar las técnicas de detección (Radeloff et al. 1999). Estudios de defoliación causada por insectos en bosques de coníferas en Quebec, Canadá, determinaron que las imágenes SPOT VGT de $1 \mathrm{~km}$ de resolución fueron efectivas para mapear mortalidad del bosque a gran escala, sin embargo, se cometieron grandes errores de comisión (Fraser y Latifovic 2004). Estudios con sensores remotos realizados en áreas montañosas de Nepal permitieron discriminar adecuadamente la vegetación boscosa de cultivos y pasturas, sin embargo no fue posible discriminar entre distintas clases de bosque (bosques maduros, bosques sucesionales y plantaciones) dado el gran error en la clasificación (Millette et al. 1995).

Aun cuando los valores medios de los niveles digitales de las áreas de entrenamiento correspondientes a mal del ciprés y a bosque de $A$. chilensis asintomático ralo difirieron significativamente para la banda 4 de la imagen SPOT, los chequeos a campo evidenciaron dificultades en la discriminación entre ambos tipos de vegetación. Para lograr un mapa de la disposición de los parches de A. chilensis con síntomas de defoliación y mortalidad con un nivel aceptable de error debió recurrirse a un método iterativo entre procesamiento de imagen y chequeo a campo. El mapa obtenido en este estudio permite cuantificar, por primera vez, la importancia del mal del ciprés, evidenciando en el área de estudio un $24 \%$ de la superficie del bosque con síntomas de la enfermedad. Las observaciones de campo proporcionadas por numerosos estudios sugerían que el mal del ciprés tenía una distribución significativa (Hranilovic 1988, Baccalá et al. 1998, Filip y Rosso 1999), no obstante se desconocía su magnitud a escala de paisaje.

En una evaluación preliminar del mapa se observa que los parches de árboles con síntomas de defoliación y mortalidad no están agrupados y limitados a un sector en particular sino que están presentes en toda el área analizada. 
A priori, no hay evidencias que sugieran la existencia de un avance continuo y radial desde un centro de dispersión. Los tamaños de los parches son muy variables en las tres categorías de vegetación analizadas. Los parches de bosque sintomático fueron numerosos y pequeños, en comparación con los parches de A. chilensis asintomático (cuadro 1). Los tamaños máximos de parche variaron desde 7,79 ha para el bosque sintomático hasta 249,5 ha para el bosque asintomático. El estudio exhaustivo de la disposición de los parches con síntomas de mal del ciprés y la cuantificación de la configuración del patrón espacial (Matteucci 1998, Li y Wu 2004) serán evaluados en futuros estudios a partir del mapa generado en este trabajo.

El mapa generado permitirá también identificar la relación entre la ocurrencia del mal del ciprés y variables ambientales (clima, topografía, suelo) a escala de paisaje (Marsden y Fielding 1999, Cowley et al. 2000, Schadt et al. 2002). Este tipo de análisis podrá ser la base para la generación de mapas de riesgo de ocurrencia de la enfermedad, los cuales han constituido una herramienta fundamental para la determinación de pautas de manejo de otros bosques (Meentemeyer et al. 2004, Van Staden 2004, Fernández y Solla 2006).

El estudio con imágenes satelitales SPOT no resultó por sí solo adecuado para la detección de los parches de A. chilensis con defoliación y mortalidad, sino que debió agregarse un gran esfuerzo de chequeo y corrección a campo. A pesar de la necesidad de un control intensivo a campo, el empleo de las imágenes satelitales redujo el tiempo, presupuesto y esfuerzo para alcanzar el objetivo. A fin de disminuir los requerimientos de control a campo podría evaluarse la utilización de otro tipo de imágenes, con mayor resolución espacial y/o espectral.

Si bien la metodología utilizada en este estudio podría aplicarse para caracterizar la distribución del mal del ciprés en otras áreas, al hacerlo deberían realizarse intensos controles de terreno. Los resultados evidenciaron que las imágenes SPOT permiten una adecuada discriminación de bosques densos de A. chilensis y bosques sintomáticos, de manera que la aproximación lograda mediante esta metodología sería más adecuada para áreas donde el mal del ciprés aparece en masas de bosque asintomático denso.

Es importante destacar que, si bien estudios patológicos previos han evidenciado que en todo el Valle 16 de Octubre el mal del ciprés comienza por el sistema radical (i.e., asociado a Phytophthora) (Rajchenberg et al. 1998, Greslebin et al. 2005), no puede descartarse que el mapa temático logrado incluya áreas afectadas donde el pulgón Cinara cupressi esté involucrado en la sintomatología de defoliación y muerte (Baldini et al. 2004).

\section{CONCLUSIONES}

En el Valle 16 de Octubre, provincia de Chubut, Argentina, la distribución de A. chilensis representa el $9 \%$ de la superficie total de los bosques puros de esta especie, constituyendo así un área representativa. El bosque afectado por mal del ciprés abarcó una superficie de 1.678 ha, que equivalen al $24 \%$ de la superficie total de A. chilensis en el área analizada. Es la primera vez que se logra cuantificar la magnitud del mal del ciprés.

Para realizar la clasificación fue necesario usar una estrategia en aproximaciones sucesivas, dada la variabilidad en los niveles de incidencia de la enfermedad y la dificultad en la separación de los parches sintomáticos de asintomáticos en situaciones de bosque ralo. A pesar de la necesidad de un control intensivo a campo, el empleo de las imágenes satelitales reduce el tiempo, presupuesto y esfuerzo para alcanzar el objetivo. Probablemente el trabajo se simplificaría, es decir, la clasificación supervisada sería más exacta y demandaría menor intensidad de trabajo de campo si se dispusiera de imágenes de mayor resolución espacial o espectral.

El mapa de la disposición espacial de parches de A. chilensis con síntomas de defoliación y mortalidad generado en este estudio servirá de base para futuros trabajos. El enfoque paisajístico resultará apropiado para hallar asociaciones entre la enfermedad y características locales del ambiente físico-biótico. De este modo, podrían desarrollarse mapas de riesgo en función del ambiente y proponer medidas de mitigación de la dispersión de la enfermedad.

\section{AGRADECIMIENTOS}

Se agradece a Juan Monjes, Darío Arquero, Marcos Menger, Luis Epele y Danilo Hernández Otaño por su asistencia en las tareas de campo, al personal del Laboratorio SIG del CIEFAP y al Dr. Thomas Kitzberger por su asesoramiento. El Prof. Miguel Calderón de la UNPSJB facilitó el uso del software ERDAS Imagine 8.4 y el ingeniero forestal Horacio Claverie de la DGByP proporcionó el acceso al software ArcView 3.2. A ambos les expresamos nuestro agradecimiento. Las imágenes SPOT fueron obtenidas a través de un subsidio de investigación de Volkswagen Foundation.

Este estudio fue financiado por el Consejo Nacional de Investigaciones Científicas y Técnicas de Argentina (CONICET) (PIP 5660-Dra A. Greslebin).

\section{REFERENCIAS}

Allen TR, DW Wong. 2006. Exploring GIS, spatial statistics and remote sensing for risk assessment of vector-borne diseases: a West Nile virus example. Int. J. Risk Assessment and Management 6: 253-275.

Baccalá N, P Rosso, M Havrylenko. 1998. Austrocedrus chilensis mortality in the Nahuel Huapi National Park (Argentina). Forest Ecology and Management 109: 261-269. 
Baldini A, A Sartori, J Aguayo. 2004. Detección del pulgón del ciprés, Cinara cupressi (Homoptera: Aphididae). Informe técnico. Programa de Protección Fitosanitaria. Chile. 11 p. Formato digital.

Barroetaveña C, M Rajchenberg. 1996. Hongos Aphyllophorales (Basidiomycetes) que causan pudriciones en Austrocedrus chilensis en pie. Boletín de la Sociedad Argentina de Botánica 31: 201-216.

Bava JO, H Gonda. 1993. Propuesta silvícola preliminar de manejo para ciprés de la cordillera. Actas Congreso Forestal Argentino y Latinoamericano, Paraná, Entre Ríos, Argentina. $11 \mathrm{p}$.

Bran D, A Pérez, D Barrios, M Pastorino, J Ayesa. 2002. Ecoregión valdiviana: distribución actual de los bosques de "ciprés de la cordillera" (Austrocedrus chilensis) - Escala 1:250.000. INTA, APN, FVSA. Bariloche.

Brockhaus JA, S Khorram, RI Bruck, MV Campbell. 1992. A comparison of Landsat TM and SPOT HRV data for use in the development of forest defoliation models. International Journal of Remote Sensing 13: 3235-3240.

Calí SG. 1996. Austrocedrus chilensis: estudio de los anillos de crecimiento y su relación con la dinámica del "mal del ciprés" en el P.N. Nahuel Huapi, Argentina. Tesis de Licenciatura en Cs. Biológicas. Bariloche, Argentina. Universidad Nacional del Comahue. 143 p.

Cano F, R Navarro Cerrillo, A García-Ferrer, M Sánchez de la Orden. 2005. Evaluación de la defoliación mediante imágenes IKONOS en masas de Quercus suber L, en el sur de España. Investigación Agraria Sistemas y Recursos Forestales 15(2): 242-252.

Carabelli F, H Claverie, S Antequera, R Scoz. 2003. Changes on landscape heterogeneity of "ciprés de la cordillera" forests. 31 p. (Second annual report to the Volkswagen Foundation).

Carabelli F. 2004. Quantitative analysis of forest fragmentation in Patagonia, Argentina. In Proceedings of the IUFRO 2004 International Workshop of Landscape Ecology: Conservation and Management of Fragmented Forest Landscapes. Tsukuba, Ibaraki, Japan, p. 83-87.

Carabelli F, H Claverie. 2005. Análisis de cambios en los paisajes de bosques de ciprés de la cordillera en el noroeste de la Provincia de Chubut. In Libro de Actas de la I Reunión sobre ecología, conservación y uso de los bosques de ciprés de la cordillera, Esquel, Chubut, Argentina. p. 61-66.

Cowley MJ, RJ Wilson, JL León Cortés, D Gutiérrez, CR Bulman, CD Thomas. 2000. Habitat-based statistical models for predicting the spatial distribution of butterflies and dayflying moths in a fragmented landscape. Journal of Applied Ecology 37: 60-72.

Dezzotti A, L Sancholuz. 1991. Los bosques de Austrocedrus chilensis en Argentina: ubicación, estructura y crecimiento. Bosque 12(1): 43-52.

Díaz-Vaz J. 1985. Austrocedrus chilensis. Descripción anatómica. Bosque 6(1): 49-50.

El Mujtar V, E Andenmatten. 2007. "Mal del ciprés": búsqueda de la causa más probable de daño mediante un análisis deductivo y comparativo. Bosque 28(1): 3-9.

Fernández JM, A Solla. 2006. Mapas de riesgo de aparición y desarrollo de la enfermedad del marchitamiento de los pinos (Bursaphelenchus xylophilus) en Extremadura. Investigación Agraria Sistemas y Recursos Forestales 15: 141-151.
Filip G, P Rosso. 1999. Cypress mortality (mal del ciprés) in the Patagonian Andes: comparisons with similar forest diseases and declines in North America. European Journal of Forest Pathology 29: 89-96.

Foody GM. 2002. Status of land cover classification accuracy assessment. Remote Sensing of Environment 80: 185-201.

Fraser RH, R Latifovic. 2005. Mapping insect-induced tree defoliation and mortality using coarse spatial resolution satellite imagery. International Journal of Remote Sensing 26: 193-200.

Greslebin A, E Hansen, L Winton, M Rajchenberg. 2004. "Mal del ciprés" (Austrocedrus chilensis decline) in Patagonia: are Phytophthora species involved? In Proceedings $3^{\text {rd }}$ Workshop of IUFRO Working Party 7.02.09 "Phytophthora in forests and natural ecosystems". Freising, Germany. p. 33.

Greslebin A, E Hansen, L Winton, M Rajchenberg. 2005. Phytophthora species from declining Austrocedrus chilensis forests in Patagonia, Argentina. Mycologia 97: 218-228.

Greslebin A, E Hansen. 2007. La causa del "mal del ciprés". Patagonia Forestal 13(1): 5-8.

Greslebin A, E Hansen, W Sutton. 2007. Phytophthora austrocedrae sp. nov., a new species associated with Austrocedrus chilensis mortality in Patagonia (Argentina). Mycological Research 11(3): 308-316.

Havrylenko M, P Rosso, S Fontenla. 1989. Austrocedrus chilensis: contribución al estudio de su mortalidad en Argentina. Bosque 10(1): 29-36.

Hranilovic S. 1988. Informe histórico sobre el mal del ciprés de la cordillera (Austrocedrus chilensis). Revista de la Asociación Forestal Argentina 42: 58-62.

Joria P, S Ahearn, M Connor. 1991. A comparison of the SPOT and Landsat Thematic Mapper satellite systems for detecting gypsy moth defoliation in Michigan. Phogrammetric Engineering and Remote Sensing 57: 16: 05-12.

Karszenbaum H. 1998. Procesamiento de imágenes satelitales para la gestión ambiental. In: Matteucci SD, GD Buzai ed. Sistemas ambientales complejos: herramientas de análisis espacial. Eudeba. Buenos Aires, Argentina. p. 197-217.

Kaya S, TJ Pultz, CM Mbogo, JC Beier, E Mushinzimana. 2002. The use of radar remote sensing for identifying environmental factors associated with malaria risk in coastal Kenya. Canada Centre for Remote Sensing. Consultado 11 Sept. 2007. Disponible en www.pcigeomatics.com/services/support_center/ tech_papers/igarss02_kaya_paper.pdf.

La Manna L. 2005. Caracterización de los suelos bajo bosque de Austrocedrus chilensis a través de un gradiente climático y topográfico en Chubut, Argentina. Bosque 26(2): 137-153.

La Manna L, F Carabelli. 2005. Distribución espacial de la declinación de los bosques de Austrocedrus chilensis en el Valle 16 de Octubre (Chubut, Argentina). In Actas Primeras Jornadas Argentinas de Ecología de Paisaje, Buenos Aires, 2 al 4 de noviembre de 2005. Formato digital.

La Manna L, M Rajchenberg. 2004a. The decline of Austrocedrus chilensis forests in Patagonia, Argentina: soil features as predisposing factors. Forest Ecology and Management 190: 345-357.

La Manna L, M Rajchenberg. 2004b. Soil properties and Austrocedrus chilensis forest decline in Central Patagonia, Argentina. Plant and Soil 263: 29-41. 
La Manna L, J Bava, M Collantes, M Rajchenberg. 2006. Características estructurales de los bosques de Austrocedrus chilensis afectados por "mal del ciprés" en Patagonia, Argentina. Bosque 27(2): 135-145.

La Manna L, M Collantes, J Bava, M Rajchenberg. Seedling recruitment of Austrocedrus chilensis in relation to cattle use, microsite environment and forest disease. Ecología Austral (En prensa).

Lencinas JD, MF Farías. 2005. Native forest mapping in Patagonian Andes based on optical satellite imagery. Photogrammetrie 2/2005: 147-156.

$\mathrm{Li} \mathrm{H,} \mathrm{J} \mathrm{Wu.} \mathrm{2004.} \mathrm{Use} \mathrm{and} \mathrm{misuse} \mathrm{of} \mathrm{landscape} \mathrm{indices.} \mathrm{Landscape}$ Ecology 19: 389-399.

Loguercio G, P Burschel, M Rey. 1999. El bosque de ciprés de la cordillera: su conservación y uso. Centro de Investigación y Extensión Forestal Andino Patagónico, Esquel, Argentina. Folleto de divulgación $\mathrm{N}^{\circ} 14.21 \mathrm{p}$.

Marsden S, A Fielding. 1999. Habitat associations of parrots on the Wallacean islands of Buru, Seram and Sumba. Journal of Biogeography 26: 439-446.

Matteucci SD. 1998. La cuantificación de la estructura del paisaje. In Matteucci SD, GD Buzai ed. Sistemas ambientales complejos: herramientas de análisis espacial. Eudeba. Buenos Aires, Argentina. p. 271-291.

Meentemeyer R, D Rizzo, W Mark, E Lotz. 2004. Mapping the risk of establishment and spread of sudden oak death in California. Forest Ecology and Management 200: 195-214

Millette TL, AR Tuladhar, RE Kasperson, BL Turner II. 1995. The use and limits of remote sensing for analysing environmental and social change in the Himalayan Middle Mountains of Nepal. In Global Environmental Change, Vol. 5. Elsevier. Gran Bretaña. p. 367-380.

Pastorino M, M Fariña, D Bran, L Gallo. 2006. Extremos geográficos de la distribución natural de Austrocedrus chilensis (Cupressaceae). Boletín de la Sociedad Argentina de Botánica 41: 307-311.

Pisek J, MJ Chen, F Deng. 2007. Assessment of a global leaf area index product from SPOT-4 VEGETATION data over selected sites in Canada. Canadian Journal of Remote Sensing 33: 341-356.

Rabassa J, O Martínez, E Getar. 2001. Geomorfología del segmento de Cordillera Patagónica y Patagonia Extrandina entre los $43^{\circ}$ y $44^{\circ}$ Lat. Sur, Prov. de Chubut. Universidad Nacional de la Patagonia San Juan Bosco. 35 p. (Informe Final PI $\mathrm{N}^{\circ} 215$ ).

Radeloff VC, DJ Mladenoff, MS Boyce. 1999. Detecting jack pine budworm defoliation using spectral mixture analysis: separating effects from determinants. Remote Sensing of Environment 69: 156-169.

Rajchenberg M, C Barroetaveña, P Cwielong, M Rossini, D Cabral, A Sívori. 1998. Fungal species associated with the decline of Austrocedrus chilensis in Patagonia, Argentina: preliminary results. In Delatour L, J Guillaumin, B Lung-Escarmant, B Marçais ed. Actas IX International Conference on Root \& Butt Rots, Carcans, France. Les Colloques 89: 235-244.

Ramsey F, D Schafer. 1997. The Statistical Sleuth: a course in methods of data analysis. Duxbury Press, Belmond, USA. $742 \mathrm{p}$.

Relva M, T Veblen. 1998. Impacts of introduced large herbivores on Austrocedrus chilensis forests in northern Patagonia, Argentina. Forest Ecology and Management 108: 27-40.

Rosso P, M Baccalá, M Havrylenko, S Fontenla. 1994. Spatial pattern of Austrocedrus chilensis wilting and the scope of autocorrelation analysis in natural forests. Forest Ecology and Management 67: 273-279.

Schadt S, F Knauer, P Kaczensky, E Revilla, T Wiegand, L Trepl. 2002. Rule-based assessment of suitable habitat and patch connectivity for the Eurasian lynx. Ecological Applications 12: $1469-1483$.

SAyDS (Secretaría de Ambiente y Desarrollo Sustentable, AR). 2005. Primer inventario de bosques nativos. Proyecto Bosques Nativos y Áreas Protegidas. (Informe Regional Bosque Andino Patagónico). 104 p.

SRNyAH (Secretaría de Recursos Naturales y Ambiente Humano, AR). 1994. Anuario de Estadística Forestal. Presidencia de la Nación. Bs.As., Argentina. 112 p.

Shurtleff MC, CW Averre III. 1997. The Plant Disease Clinic and Field Diagnosis of Abiotic Diseases. APS Press, St. Paul, USA. 245 p.

Smits PC, SG Dellepiane, RA Schowengerdt. 1999. Quality assessment of image classification algorithms for landcover mapping: a review and a proposal for a cost-based approach. International Journal of Remote Sensing 20(8): 1461-1486.

Towers P, A von Martini. 2002. Conceptos iniciales sobre Teledetección y su aplicación al agro. 138 p. Consultado 22 may. 2007. Disponible en http://www.elsitioagricola. com/Soft/agrisat/libroTeledeteccion.asp

Van Staden V, B Erasmus, J Roux, M Wingfield, A Van Jaarsveld. 2004. Modelling the spatial distribution of two important South African plantation forestry pathogens. Forest Ecology and Management 187: 61-73

Varsavsky E, L Bettucci, D Rodríguez, C Gómez,. 1975. Observaciones preliminares sobre la mortalidad del ciprés (Austrocedrus chilensis) en los bosques patagónicos, Bariloche, Argentina. 11 p. (Publicación Fundación Bariloche $\mathrm{N}^{\mathrm{o}}$ 19).

Veblen T, B Burns, T Kitzberger, A Lara, R Villalba. 1995. The ecology of the conifers of southern South America. In Enright N, R Hill ed. Ecology of the southern conifers. Melbourne University Press, Melbourne, Australia. p. 120-155.

Veblen T, T Kitzberger, B Burns, A Rebertus. 1996. Perturbaciones y dinámica de regeneración en bosques andinos del sur de Chile y Argentina. In Armesto J, C Villagrán, M Arroyo ed. Ecología de los bosques nativos de Chile. Santiago de Chile, Chile. Editorial Universitaria. p. 169-198. 